\title{
Values of polynomials with integer coefficients and distance to their common zeros
}

\author{
by
}

\author{
Francesco Amoroso (Pisa)
}

1. Introduction. Let $f_{1}, \ldots, f_{m} \in \mathbb{Z}\left[x_{1}, \ldots, x_{n}\right]$ be polynomials of maximum degree $D$ and height (= maximum absolute value of the coefficients) $\leq H$ defining an affine variety $\mathbb{V} \subset \mathbb{C}^{n}$ of codimension $k$. Denote by dist the distance in $\mathbb{C}^{n}$ with respect to the norm $|\omega|=\max _{i}\left|\omega_{i}\right|$. In $[\mathrm{B}]$ W. D. Brownawell proved the following inequality of Łojasiewicz type:

For any $\omega \in \mathbb{C}^{n}$ we have

$$
\min \{\operatorname{dist}(\omega, \mathbb{V}), 1\}^{(n+1)^{2}} \leq C_{1}^{D}\left(H \max \{1,|\omega|\}^{2}\right)^{C_{2}} \max _{i}\left|f_{i}(\omega)\right|^{D^{-n}}
$$

where $C_{1}=\exp \left\{11(n+1)^{5}\right\}$ and $C_{2}=(n+1)^{2}$.

This result is essentially the best possible, except perhaps for the values of the constants and for the exponent $(n+1)^{2}$ in the left hand side. S. Ji, J. Kollár and B. Shiffman [J-K-S] have recently proved a similar result for polynomials over a field of arbitrary characteristic without this exponent but with an ineffective dependence on the coefficients. In spite of that, we can look for other relations between the values of the $f_{i}$ 's and the distance to their common zeros in $\mathbb{C}^{n}$. For a polynomial $f \in \mathbb{Z}\left[x_{1}, \ldots, x_{n}\right]$ we denote its size (= degree + logarithmic height) by $t(f)$; for $\alpha \in \mathbb{C}^{n}$ we also denote by $t(\alpha)$ the minimum size of a non-zero polynomial $f \in \mathbb{Z}\left[x_{1}, \ldots, x_{n}\right]$ for which $f(\alpha)=0$ (if there are no such polynomials we put $t(\alpha)=\infty$ ). In this paper we deal with the following problem:

Let $\omega$ be in the unit ball of $\mathbb{C}^{n}$ and suppose that

$$
\max _{i}\left|f_{i}(\omega)\right|<\exp \left\{-C\left\{\max _{i} t\left(f_{i}\right)\right\}^{\tau}\right\}
$$

for some $C$ greater than a constant $A=A(n)$ and for some $\tau \geq n+1$. Find the best value $\eta=\eta(\tau, n, k)$ for which there exist constants $e=e(n, k)$ and

1991 Mathematics Subject Classification: Primary 11J25; Secondary 11J82. 
$B=B(n)$ such that

$$
\min _{\substack{\alpha \in \mathbb{C}^{n} \\ f_{i}(\alpha)=0}}|\alpha-\omega|<\exp \left\{-B^{-1} C^{e} t(\alpha)^{\eta}\right\} .
$$

Roughly speaking, we are looking for an upper bound for transcendence measures in terms of approximation measures (for definitions see [P2]). If $n=1$, this problem is completely solved: we can take $\eta=\tau$. In the general case, only partial results are known. For example, using a theorem of P. Philippon, it is easy to see that we can choose $\eta=\tau-n$ (here $-n$ corresponds to $D^{-n}$ in Brownawell's inequality), and we conjecture that this exponent can be replaced by $\tau$. In the present paper we prove this in three special cases: if $\tau=n+1$, if $\mathbb{V}$ is discrete, or if $n=2$. Our first result is the following theorem:

Theorem 1. For any integer $n \geq 1$ there exist two constants $A, B>0$ having the following property. Let $f_{1}, \ldots, f_{m}$ and $\omega$ be as before and assume that (1) holds for some $\tau \geq n+1$ and some $C>A$. Then, if the affine variety $\mathbb{V}$ defined by the $f_{i}$ 's has codimension $k$, we can find $\alpha \in \mathbb{V}$ such that (2) holds with

$$
\eta=\max \left\{n+1+\frac{\tau-(n+1)}{n+1-k}, \tau-n\right\}
$$

and

$$
e= \begin{cases}1 & \text { if } \eta=\tau-n, \\ 2^{-n+k} & \text { otherwise. }\end{cases}
$$

Notice that $\eta=\tau$ if $\tau=n+1$ or if $k=n$ (i.e. if $\mathbb{V}$ is discrete).

The case $m=1$ is of particular interest. First of all, Theorem 1 allows us to give a positive answer to the following conjecture of G. V. Chudnovsky (see [C], Problem 1.3, p. 178):

For any integer $n \geq 1$ there exists a positive constant $C$ such that for almost all $\omega$ in the unit ball of $\mathbb{C}^{n}$ (in the sense of the Lebesgue measure in $\mathbb{R}^{2 n}$ ) the inequality $\log |f(\omega)| \leq-C t(f)^{n+1}$ has only finitely many solutions $f \in \mathbb{Z}\left[x_{1}, \ldots, x_{n}\right]$.

Indeed, it is easy to see that for any $n \in \mathbb{N}$ there exists a positive constant $C$ such that the set of $\omega$ 's in the unit ball of $\mathbb{C}^{n}$ for which the inequality

$$
|\alpha-\omega|<\exp \left\{-C t(\alpha)^{n+1}\right\}
$$

has infinitely many solutions $\alpha \in \mathbb{C}^{n}$ is negligible for the Lebesgue measure (see the proof of $[\mathrm{A}]$, Proposition 5). Using Theorem 1, we immediately obtain Chudnovsky's conjecture.

Moreover, for $m=1$ and $n \geq 2$, (3) can be easily improved to

$$
\eta=\max \left\{n+\frac{\tau-2}{n-1}, \tau-1\right\}
$$


(see Theorem 2 in $\S 3$ ), which implies the full conjecture $\eta=\tau$ for $n=2$. On the other hand, in $[\mathrm{A}]$ we proved (in a slightly weaker form) that we can choose for $\eta$ the maximum between $\tau-2+\tau / n$ and the positive root of $x^{2}+(1-\tau) x+n-1-\tau=0$. This result approaches our conjecture for $\tau \rightarrow \infty$, but, unfortunately, the proof given in [A] contains some minor errors. In the appendix we shall give a proof of the slightly weaker result

$$
\eta>0, \quad \eta^{2}+(1-\tau) \eta+n-\tau=0
$$

(which also approaches our conjecture) and corrections of other mistakes which occur in $[\mathrm{A}]\left({ }^{1}\right)$.

2. Technical results. For the proofs, we use the theory of Chow forms, as developed by Yu. V. Nesterenko (see [N1], [N2] and [N3]) and by P. Philippon (see [P1] and [P2]). We briefly summarize the notations employed by Nesterenko. Given a homogeneous unmixed ideal $I$ of rank $n+1-r$ in the ring $\mathbb{Z}\left[x_{0}, \ldots, x_{n}\right]$ having Chow form $F=F\left(u^{1}, \ldots, u^{r}\right) \in \mathbb{Z}\left[u_{0}^{1}, \ldots, u_{n}^{r}\right]$, we denote by $H(I)$ the maximum absolute value of the coefficients of $F$, by $N(I)$ the degree of $F$ with respect to $u_{0}^{1}, \ldots, u_{n}^{1}$, and by $t(I)$ the number $N(I)+\log H(I)$. Given $\omega^{\prime}$ in the projective space $\mathbb{P}^{n}$ over $\mathbb{C}$, we define $|I|_{\omega^{\prime}}$ as

$$
|I|_{\omega^{\prime}}=H(\kappa(F)) /\left|\omega^{\prime}\right|^{r N(I)},
$$

where $H(\kappa(F))$ is the maximum absolute value of the coefficients of the polynomial

$$
\kappa(F) \in \mathbb{C}\left[s_{j, k}^{i}\right]_{\substack{i=1, \ldots, r \\ 0 \leq j<k \leq n}}
$$

obtained by replacing in $F$ the vectors $u^{i}$ by $S^{i} \omega^{\prime}$, with $S^{i}(i=1, \ldots, r)$ being skew-symmetric matrices in the new variables $s_{j, k}^{i}(0 \leq j<k \leq n)$. For more details, see [N3] (Nesterenko uses the notation $\left|I\left(\omega^{\prime}\right)\right|$ instead of $\left.|I|_{\omega^{\prime}}\right)$. Given a homogeneous polynomial $Q \in \mathbb{Z}\left[x_{0}, \ldots, x_{n}\right]$ and $\omega^{\prime} \in \mathbb{P}^{n}$ we let

$$
|Q|_{\omega^{\prime}}=\left|Q\left(\omega^{\prime}\right)\right| /\left|\omega^{\prime}\right|^{\operatorname{deg} Q} .
$$

We start with an easy consequence of the box principle.

Lemma 1. Let $n \geq 1$ be an integer and let $\omega^{\prime} \in \mathbb{P}^{n}$. Then there exist two positive constants $c_{1}$ and $c_{2}$ depending only on $n$ such that for any real number $N>c_{1}$ there exists a non-zero homogeneous polynomial $Q \in$ $\mathbb{Z}\left[x_{0}, \ldots, x_{n}\right]$ with size $\leq N$ satisfying

$$
|Q|_{\omega^{\prime}} \leq \exp \left\{-c_{2} N^{n+1}\right\} .
$$

Proof. Let $H$ and $d$ be two positive integers and let $\Lambda$ be the set of homogeneous polynomials $Q \in \mathbb{Z}\left[x_{0}, \ldots, x_{n}\right]$ of degree $d$ with non-negative

$\left({ }^{1}\right)$ I am grateful to Yuriǔ Nesterenko who drew my attention to these mistakes. 
coefficients bounded by $H$. This set has cardinality $(H+1)^{D}, D=\left(\begin{array}{c}d+n \\ n\end{array}\right)$, and for any $Q \in \Lambda$ we have $|Q|_{\omega^{\prime}} \leq D H$. Let

$$
\delta=\min _{Q_{1}, Q_{2} \in \Lambda, Q_{1} \neq Q_{2}}\left|Q_{1}-Q_{2}\right|_{\omega^{\prime}} .
$$

The ball in $\mathbb{C}$ with centre at the origin and radius $D H+\delta / 2$ contains the disjoint union of the open balls of centre $Q\left(\omega^{\prime}\right)\left|\omega^{\prime}\right|^{-d}(Q \in \Lambda)$ and radius $\delta / 2$. This gives

$$
\delta \leq \frac{2 D H}{(H+1)^{D}-1} \leq 2 D H^{1-D}
$$

and so there exist two polynomials $Q_{1}, Q_{2} \in \Lambda, Q_{1} \neq Q_{2}$, such that

$$
\left|Q_{1}-Q_{2}\right|_{\omega^{\prime}} \leq 2 D H^{1-D} \text {. }
$$

The polynomial $Q=Q_{1}-Q_{2}$ has degree $d$, height (= maximum absolute value of the coefficients $) \leq H$ and satisfies $|Q|_{\omega^{\prime}} \leq 2 D H^{1-D}$. The lemma follows upon taking $d=[N / 2]$ and $H=[\exp \{N / 2\}]$.

Given $\omega^{\prime}, \alpha^{\prime}$ in the complex projective space $\mathbb{P}^{n}$, we put

$$
d\left(\alpha^{\prime}, \omega^{\prime}\right)=\frac{\max _{0 \leq i<j \leq n}\left|\omega_{i}^{\prime} \alpha_{j}^{\prime}-\omega_{j}^{\prime} \alpha_{i}^{\prime}\right|}{\max _{0 \leq i \leq n}\left|\alpha_{i}^{\prime}\right| \max _{0 \leq i \leq n}\left|\omega_{i}^{\prime}\right|} .
$$

Remark. Let $\omega^{\prime}=(1, \omega)$ where $\omega$ is in the unit ball of $\mathbb{C}^{n}$ and assume $d\left(\alpha^{\prime}, \omega^{\prime}\right)<1$. Then $\alpha_{0}^{\prime} \neq 0$ and the vector $\alpha \in \mathbb{C}^{n}$ defined by $\alpha_{i}=\alpha_{i}^{\prime} / \alpha_{0}^{\prime}(i=$ $1, \ldots, n)$ satisfies $|\alpha-\omega| \leq \max \{1,|\alpha|\} d\left(\alpha^{\prime}, \omega^{\prime}\right)$. This gives $\max \{1,|\alpha|\} \leq$ $\left(1-d\left(\alpha^{\prime}, \omega^{\prime}\right)\right)^{-1}$ and so

$$
|\alpha-\omega| \leq \frac{d\left(\alpha^{\prime}, \omega^{\prime}\right)}{1-d\left(\alpha^{\prime}, \omega^{\prime}\right)}
$$

In particular, if $d\left(\alpha^{\prime}, \omega^{\prime}\right) \leq 1 / 2$, we have $|\alpha-\omega| \leq 2 d\left(\alpha^{\prime}, \omega^{\prime}\right)$.

Lemma 2. For any integer $n \geq 1$ there exists a constant $A>0$ having the following property. Let $k \leq n$ be a positive integer, let $\tau \geq k+1, \eta \in$ $[n+1, \tau+n-k]$ and $\theta>1$ be real numbers and let $\omega^{\prime} \in \mathbb{P}^{n}$. Assume that there exists a homogeneous prime ideal $\wp \subset \mathbb{Z}\left[x_{0}, \ldots, x_{n}\right]$ of rank $k$ such that $\wp \cap \mathbb{Z}=\{0\}$ and

$$
|\wp|_{\omega^{\prime}}<\exp \left\{-C t(\wp)^{\tau / k}\right\}
$$

for some $C \geq A \theta$. Then either there exists $\alpha^{\prime} \in \mathbb{V}_{\mathbb{P}}(\wp)$, the projective variety defined by $\wp$, such that

$$
d\left(\alpha^{\prime}, \omega^{\prime}\right)<\exp \left\{-A^{-1} \theta t\left(\alpha^{\prime}\right)^{\eta}\right\},
$$

or there exists a homogeneous prime ideal $\wp^{\prime} \subset \mathbb{Z}\left[x_{0}, \ldots, x_{n}\right]$ of rank $k+1$ such that $\wp^{\prime} \cap \mathbb{Z}=\{0\}, \wp^{\prime} \supset \wp$ and

$$
\left|\wp^{\prime}\right|_{\omega^{\prime}}<\exp \left\{-A^{-1} \theta^{-1} C t\left(\wp^{\prime}\right)^{(n+1-\eta+\tau) /(k+1)}\right\} .
$$

Moreover, if $k=n$ or if $\eta \leq \tau-k$, the first case occurs. 
Proof. Denote by $c_{3}, \ldots, c_{10}$ positive constants depending only on $k, n$, $\tau$ and $\eta$. If $\omega^{\prime} \in \mathbb{V}_{\mathbb{P}}(\wp)$ we put $\alpha^{\prime}=\omega$; otherwise let $\alpha^{\prime} \in \mathbb{V}_{\mathbb{P}}(\wp)$ be such that $\delta=d\left(\omega^{\prime}, \alpha^{\prime}\right)>0$ is minimal. Using Lemma 6 of [N3], we see that

$$
-\delta>C t(\wp)^{(\tau-k) / k}-c_{3} .
$$

Moreover, Corollary 3 of [N1] gives

$$
t\left(\alpha^{\prime}\right) \leq c_{4} t(\wp)^{1 / k}
$$

Hence

$$
-\delta>\left(C c_{4}^{-\tau+k}-c_{3}\right) t\left(\alpha^{\prime}\right)^{\tau-k} \geq A^{-1} \theta t\left(\alpha^{\prime}\right)^{\eta}
$$

provided that $\eta \leq \tau-k$ and $A$ is sufficiently large. Now assume $\eta>\tau-k$ and put

$$
N=\theta^{-y} t(\wp)^{-x}(-\delta)^{y}
$$

where

$$
x=\frac{\eta-(n+1)+\tau / k}{\eta+(n+1) k-\tau}>0 \quad \text { and } \quad y=\frac{k+1}{\eta+(n+1) k-\tau} \geq 1 / n .
$$

From (4) and from $\eta \leq \tau+n-k$ we obtain

$$
\begin{aligned}
N & \geq \theta^{-y} t(\wp)^{-x}\left(C t(\wp)^{\tau / k-1}-c_{1}\right)^{y} \\
& \geq \theta^{-y}\left(C-c_{1}\right)^{y} t(\wp)^{(\tau+n-k-\eta) /(\eta+(n+1) k-\tau)} \geq c_{1}
\end{aligned}
$$

provided that $A$ is sufficiently large. Therefore, Lemma 1 gives a non-zero homogeneous polynomial $Q \in \mathbb{Z}\left[x_{0}, \ldots, x_{n}\right]$ which satisfies

$$
\begin{aligned}
t(Q) & \leq N, \\
|Q|_{\omega^{\prime}} & \leq \exp \left\{-c_{2} N^{n+1}\right\} .
\end{aligned}
$$

We distinguish three cases:

- First case: $Q \notin \wp$ and $\mu:=c_{2} N^{n+1}(-\delta)^{-1}<1$. By (8) we have $|Q|_{\omega^{\prime}} \leq \exp \{\mu \delta\}$. If $k<n$, Lemma 4 of [N3] gives a homogeneous ideal $I \subset \mathbb{Z}\left[x_{0}, \ldots, x_{n}\right]$ of pure rank $k+1$ whose zeros coincide with the zeros of the ideal $(\wp, Q)$ and such that

$$
\begin{aligned}
t(I) & \leq c_{5} t(Q) t(\wp), \\
\log |I|_{\omega^{\prime}} & \leq \mu \log |\wp|_{\omega^{\prime}}+c_{6} t(\wp) t(Q) .
\end{aligned}
$$

Taking into account (10), (7), $\eta \leq \tau+n-k$ and (9), we get

$$
\begin{aligned}
\log |I|_{\omega^{\prime}} & \leq-c_{2} C N^{n+1}(-\delta)^{-1} t(\wp)^{\tau / k}+c_{6} t(\wp) N \\
& =-c_{2} \theta^{-1} C(t(\wp) N)^{(n+1-\eta+\tau) /(k+1)}+c_{6} t(\wp) N \\
& \leq-\left(c_{2} \theta^{-1} C-c_{6}\right)\left(c_{5}^{-1} t(I)\right)^{(n+1-\eta+\tau) /(k+1)} .
\end{aligned}
$$


Proposition 2 of [N2] gives a homogeneous prime ideal $\wp^{\prime} \in \mathbb{Z}$ of rank $k+1$ whose zeros are zeros of $I$ such that $\wp^{\prime} \cap \mathbb{Z}=\{0\}$ and

$$
\begin{aligned}
\log \left|\wp^{\prime}\right|_{\omega^{\prime}} & <-c_{7}\left(\theta^{-1} C-c_{8}\right) t\left(\wp^{\prime}\right)^{(n+1-\eta+\tau) /(k+1)} \\
& \leq-c_{9} \theta^{-1} C t\left(\wp^{\prime}\right)^{(n+1-\eta+\tau) /(k+1)}
\end{aligned}
$$

provided that $A$ is sufficiently large.

If $k=n$, the same Lemma 4 of [N3] gives $\mu \log |\wp|_{\omega^{\prime}}+c_{6} t(\wp) t(Q) \geq 0$, which cannot occur if $A$ is sufficiently large.

- Second case: $Q \notin \wp$ and $\mu \geq 1$. Taking into account (5) we obtain

$$
-\delta \geq c_{10} \theta^{(n+1) y /((n+1) y-1)} t\left(\alpha^{\prime}\right)^{k(n+1) x /((n+1) y-1)} \geq A^{-1} \theta t\left(\alpha^{\prime}\right)^{\eta}
$$

since

$$
\frac{k(n+1) x}{(n+1) y-1}-\eta=\frac{(\eta-n-1)(\eta-\tau+k(n+1))}{\tau+(n+1)-\eta} \geq 0 .
$$

- Third case: $Q \in \wp$. Using (7) and (5), we obtain

$$
t\left(\alpha^{\prime}\right) \leq t(Q) \leq \theta^{-y} t(\wp)^{-x}(-\delta)^{y} \leq c_{4}^{k x} \theta^{-y} t\left(\alpha^{\prime}\right)^{-k x}(-\delta)^{y}
$$

and

$$
-\delta \geq A^{-1} \theta t\left(\alpha^{\prime}\right)^{\eta}
$$

Our assertion comes from (6), (11), (12) and (13).

By induction we deduce the following

Proposition 1. For any integer $n \geq 1$ there exists a positive constant $B$ having the following property. Let $k \leq n$ be a positive integer and let $\omega^{\prime} \in \mathbb{P}^{n}$. Assume that there exists a homogeneous prime ideal $\wp \subset \mathbb{Z}\left[x_{0}, \ldots, x_{n}\right]$ of rank $k$ such that $\wp \cap \mathbb{Z}=\{0\}$ and

$$
|\wp|_{\omega^{\prime}}<\exp \left\{-C t(\wp)^{\tau / k}\right\}
$$

for some $C \geq B$ and some $\tau \geq n+1$. Then there exists $\alpha^{\prime} \in \mathbb{V}_{\mathbb{P}}(\wp)$ such that

$$
d\left(\alpha^{\prime}, \omega^{\prime}\right)<\left\{-B^{-1} C^{e} t\left(\alpha^{\prime}\right)^{\eta}\right\}
$$

where

$$
\eta=\max \left\{n+1+\frac{\tau-(n+1)}{n+1-k}, \tau-k\right\}
$$

and

$$
e= \begin{cases}1 & \text { if } \eta=\tau-k \\ 2^{-n+k} & \text { otherwise. }\end{cases}
$$

Pr o o f. If $\eta=\tau-k$, Lemma 2 gives our claim. Assume

$$
\eta=n+1+\frac{\tau-(n+1)}{n+1-k} .
$$


From $\tau \geq n+1$ we obtain $\eta \geq n+1$. We shall prove the proposition by induction on $k$.

- $k=n$. Lemma 2 , with $\theta=A^{-1} C$, gives $\alpha^{\prime} \in \mathbb{V}_{\mathbb{P}}(\wp)$ such that

$$
d\left(\alpha^{\prime}, \omega^{\prime}\right)<\exp \left\{-A^{-2} C t\left(\alpha^{\prime}\right)^{\eta}\right\} .
$$

- $k<n$. We apply Lemma 2 with $\theta=C^{1 / 2}$. If there exists $\alpha^{\prime} \in \wp$ such that

$$
d\left(\alpha^{\prime}, \omega^{\prime}\right)<\exp \left\{-A^{-1} C^{1 / 2} t\left(\alpha^{\prime}\right)^{\eta}\right\}
$$

our assertion follows. Otherwise, there exists a homogeneous prime ideal $\wp^{\prime} \supset \wp$ of rank $k+1$ such that $\wp^{\prime} \cap \mathbb{Z}=\{0\}$ and

$$
\left|\wp^{\prime}\right|_{\omega^{\prime}}<\exp \left\{-A^{-1} C^{1 / 2} t\left(\wp^{\prime}\right)^{\tau^{\prime} /(k+1)}\right\},
$$

with $\tau^{\prime}=n+1-\eta+\tau$. By inductive hypothesis, we can find $\alpha^{\prime} \in \wp$ with

$$
d\left(\alpha^{\prime}, \omega^{\prime}\right)<\exp \left\{-B^{-1} C^{2^{-n+k}} t\left(\alpha^{\prime}\right)^{\eta^{\prime}}\right\}
$$

where

$$
\eta^{\prime}=n+1+\frac{\tau^{\prime}-(n+1)}{n-k}=\eta
$$

Using Theorem 2 of [P2] (with $I_{N, 1}=\ldots=I_{N, k+1}=\left(Q_{N}\right)$ and the polynomial $Q_{N}$ of size $\leq N$ given by Lemma 1 as in the proof of Lemma 2) we find a result similar to the previous one but with a worse exponent:

For any integer $n$ there exist constants $A, B>0$ having the following property. Let $k \leq n$ be an integer, $\tau \geq n+1$ a real number and let $\omega^{\prime} \in \mathbb{P}^{n}$. Assume that there exists a homogeneous prime ideal $\wp \subset \mathbb{Z}\left[x_{0}, \ldots, x_{n}\right]$ of rank $k$ such that $\wp \cap \mathbb{Z}=\{0\}$ and

$$
|\wp|_{\omega^{\prime}}<\exp \left\{-A t(\wp)^{\tau / k}\right\} \text {. }
$$

Then we can find $(1, \alpha) \in \mathbb{C}^{n}$ such that

$$
d\left(\alpha^{\prime}, \omega^{\prime}\right)<\exp \left\{-B^{-1} t\left(\alpha^{\prime}\right)^{\eta}\right\}
$$

where

$$
\eta=n+1+k \frac{\tau-(n+1)}{(n+1-k) \tau} .
$$

3. Proof of the main results. We have a relation between the value of a homogeneous prime ideal $\wp$ at $\omega^{\prime} \in \mathbb{P}^{n}$ and its projective distance from the variety defined by $\wp$. Our next task is to put it in terms of polynomials.

Lemma 3. Let $P_{1}, \ldots, P_{m} \in \mathbb{Z}\left[x_{0}, \ldots, x_{n}\right]$ be non-zero homogeneous polynomials of size $\leq T$ and let $\omega^{\prime} \in \mathbb{P}^{n}$. Let $\varepsilon=\max _{i}\left|P_{i}\right|_{\omega^{\prime}}$ and assume $\varepsilon<\exp \left\{-A T^{n+1}\right\}$ where $A>0$ depends only on $n$. Then there exists 
an unmixed homogeneous ideal $J \subset \mathbb{Z}\left[x_{0}, \ldots, x_{n}\right]$ of rank $k \leq n$ such that $\sqrt{J \mathbb{Q}\left[x_{0}, \ldots, x_{n}\right]} \cap \mathbb{Z}\left[x_{0}, \ldots, x_{n}\right] \supset I=\left(P_{1}, \ldots, P_{m}\right)\left({ }^{2}\right)$ and

$$
t(J) \leq B_{1} T^{k}, \quad|J|_{\omega^{\prime}} \leq \varepsilon^{B_{2}^{-1}}
$$

where $A, B_{1}$ and $B_{2}$ are positive constants depending only on $n$.

Proof. Denote by $c_{h, 11}, \ldots, c_{h, 16}(h=1, \ldots, n+1)$ positive constants depending only on $n$. We will show by induction that for $h=1, \ldots, n+1$ there exist unmixed homogeneous ideals $J_{h} \subset \mathbb{Z}\left[x_{0}, \ldots, x_{n}\right]$ of rank $h$ such that $J_{h} \cap \mathbb{Z}=\{0\}$ (for $h \leq n$ ) and

$$
t\left(J_{h}\right) \leq c_{h, 11}^{h} T^{h}, \quad\left|J_{h}\right|_{\omega^{\prime}} \leq \varepsilon^{c_{h, 12}} .
$$

Since the last inequalities fail for $h=n+1$, our assertion will be proved.

- $h=1$. We take $J_{1}=\left(P_{1}\right)$ and we apply Proposition 1 of [N3].

- $h \Rightarrow h+1$. Assume $\left(14_{h}\right)$ satisfied for some $h \leq n$ and for some ideal $J_{h}$. We denote by $J_{h, 1}$ the intersection of the primary components of $J_{h}$ whose radical contains $I$ and by $J_{h, 2}$ the intersection of the other components. Using [N2], Proposition 2, and Gelfond's inequality [G], Lemma II, p. 135, it is easy to see that

$$
\begin{aligned}
t\left(J_{h, 1}\right) & \leq c_{h, 13} T^{h}, \quad t\left(J_{h, 2}\right) \leq c_{h, 13} T^{h}, \\
\left|J_{h, 1}\right|_{\omega^{\prime}}\left|J_{h, 2}\right|_{\omega^{\prime}} & <\varepsilon^{c_{h, 12}} \exp \left\{c_{h, 14} T^{h}\right\} \leq \varepsilon^{c_{h, 12}-c_{h, 14} / A} .
\end{aligned}
$$

Since we are assuming that our claim is wrong, we must have $\left|J_{h, 1}\right|_{\omega^{\prime}} \geq \varepsilon^{B_{2}^{-1}}$; therefore

$$
\left|J_{h, 2}\right|_{\omega^{\prime}}<\varepsilon^{c_{h, 12}-c_{h, 14} / A-1 / B_{2}} .
$$

A classical trick (see for instance [P1], Lemma 1.9) allows us to find homogeneous polynomials $a_{1}, \ldots, a_{m} \in \mathbb{Z}\left[x_{0}, \ldots, x_{n}\right]$ with $\operatorname{deg} a_{j}=\max \left(\operatorname{deg} P_{i}\right)-$ $\operatorname{deg} P_{j}(j=1, \ldots, m)$ such that $P=a_{1} P_{1}+\ldots+a_{m} P_{m}$ is not a zero-divisor on $\mathbb{Z}\left[x_{0}, \ldots, x_{n}\right] / J_{h, 2}$. Moreover, we can choose the $a_{i}$ 's in such a way that their heights are bounded by the number of irreducible components of $J_{h, 2}$ and so, a fortiori, by $c_{h, 13} T^{h}$. From this, we obtain

$$
t(P) \leq c_{h, 15} T, \quad|Q|_{\omega^{\prime}} \leq \varepsilon^{c_{h, 16}} .
$$

Using (15), (16) and the last inequalities, Proposition 3 of [N2] gives an unmixed ideal $J_{h+1} \subset \mathbb{Z}\left[x_{0}, \ldots, x_{n}\right]$ of rank $h+1$ such that inequalities $\left(14_{h+1}\right)$ hold.

Using Proposition 2 of [N2], we easily deduce

Proposition 2. For any integer $n \geq 1$ there exist two constants $A, B>0$ having the following property. Let $\tau \geq n+1$ be a real number and let $\omega^{\prime} \in \mathbb{P}^{n}$.

$\left({ }^{2}\right) \operatorname{rank}(J)$ may be greater than $\operatorname{rank}(I)$. 
Assume that there exist non-zero homogeneous polynomials $P_{1}, \ldots, P_{m} \in$ $\mathbb{Z}\left[x_{0}, \ldots, x_{n}\right]$ of size $\leq T$ such that $\max _{i}\left|P_{i}\right|_{\omega^{\prime}}<\exp \left\{-C T^{\tau}\right\}$ for some $C \geq A$. Then there exists a homogeneous prime ideal $\wp \subset \mathbb{Z}\left[x_{0}, \ldots, x_{n}\right]$ of rank $k \leq n$ such that $\wp \cap \mathbb{Z}=\{0\}, \wp \supset\left(P_{1}, \ldots, P_{m}\right)$ and

$$
|\wp|_{\omega^{\prime}}<\exp \left\{-B^{-1} C t(\wp)^{\tau / k}\right\} .
$$

Proof of Theorem 1. Let $f_{1}, \ldots, f_{m}$ be as in Theorem 1 , let $P_{i}=$ ${ }^{h} f_{i}$ be the homogenization of $f_{i}(i=1, \ldots, m)$ and let $\omega^{\prime}=(1, \omega)$. Applying Proposition 1 to the homogeneous prime ideal $\wp$ given by Proposition 2 (which has rank $\geq k$ since $x_{0} \notin \wp$ ) and using the remark before Lemma 2, we obtain our claim.

To improve the previous theorem when $m=1$, we need the following lemma of Chudnovsky (see [C], Lemma 1.1, p. 424).

Lemma 4. Let $f \in \mathbb{C}\left[x_{1}, \ldots, x_{n}\right]$ of degree $\leq d$ and let $\omega \in \mathbb{C}^{n}$. Then for any $\lambda \in \mathbb{N}^{n}$ there exists a zero $\alpha \in \mathbb{C}^{n}$ of $f$ such that

$$
\frac{1}{|\lambda| !}\left|\frac{\partial^{\lambda} f(\omega)}{\partial x^{\lambda}}\right||\alpha-\omega|^{|\lambda|} \leq 2^{d}|f(\omega)|
$$

(here $\left.|\lambda|=\lambda_{1}+\ldots+\lambda_{n}\right)$.

TheOREM 2. For any integer $n \geq 2$ there exists a constant $B>0$ having the following property. Let $f \in \mathbb{Z}\left[x_{1}, \ldots, x_{n}\right]$ of size $\leq T$ and let $\omega$ be in the unit ball of $\mathbb{C}^{n}$ such that

$$
|f(\omega)|<\exp \left\{-C T^{\tau}\right\}
$$

for some $C \geq B$ and some $\tau \geq n+1$. Then there exists $\alpha \in \mathbb{C}^{n}$ on the hypersurface $\{f=0\}$ such that

$$
|\alpha-\omega|<\exp \left\{-B^{-1} C^{e} t(\alpha)^{\eta}\right\},
$$

where

$$
\eta=\max \left\{n+\frac{\tau-2}{n-1}, \tau-1\right\}
$$

and

$$
e= \begin{cases}1 & \text { if } \eta=\tau-1 \\ 2^{-n+2} & \text { otherwise }\end{cases}
$$

Proof. We can assume $f$ irreducible and $D_{x_{1}} f=\partial f / \partial x_{1} \not \equiv 0$. Inequality (17) with $\eta=\tau-1$ and $e=1$ is easily proved applying Proposition 1 to the principal prime ideal $\wp=(f)$. Moreover, if

$$
\left|D_{x_{1}} f(\omega)\right| \geq \exp \left\{-\frac{C}{2} t(f)^{\tau}\right\}
$$


Lemma 4 gives $\alpha \in \mathbb{C}^{n}$ such that $f(\alpha)=0$ and

$$
\log |\alpha-\omega|<-\frac{C}{4} t(f)^{\tau} .
$$

In this case, (17) is proved with $\eta=\tau$ and $e=1$. Otherwise, using Proposition 2 with $P_{1}={ }^{h} f$ and $P_{2}={ }^{h} D_{x_{1}} f$, we can find a homogeneous prime ideal $\wp \subset \mathbb{Z}\left[x_{1}, \ldots, x_{n}\right]$ of rank $\geq 2$ (actually $=2$ ), containing the ideal $\left({ }^{h} f,{ }^{h} D_{x_{1}} f\right)$, such that $|\wp|_{\omega^{\prime}}<\exp \left\{-c_{17} C t(\wp)^{\tau / 2}\right\}$. Proposition 1 and the remark before Lemma 2 give (17) with

$$
\eta=n+1+\frac{\tau-(n+1)}{n-1}=n+\frac{\tau-2}{n-1}
$$

and $e=2^{-n+2}$.

Appendix: Corrections to "Polynomials with high multiplicity" (Acta Arith. 56 (1990), 345-364). In this section we refer to lemmas, propositions, theorems, numbers of equations and lines of the paper [A] using italic type.

The inequalities (5) on $p .354$ are not true. More precisely, define for $k=1, \ldots, k_{0}$ and $j=1, \ldots, s_{k}$,

$$
\Lambda_{j k}=\mathbb{V}_{\mathbb{P}}\left(\wp_{j, h}\right) \backslash \bigcup_{h=1}^{k-1} \bigcup_{j=1}^{s_{h}} \mathbb{V}_{\mathbb{P}}\left(\wp_{j, h}\right),
$$

where the symbols have the same meaning as in [A]. Lemma 4 on p. 354 gives

$$
i_{\omega}\left(J_{k}\right) \geq \prod_{h=0}^{k-1}\left(t_{k} M-t_{h} M\right) \quad \text { for any } \omega \in \Lambda_{j k} .
$$

If $\Lambda_{j k}$ is not empty, it is a non-empty Zariski open set in $\mathbb{V}_{\mathbb{P}}\left(\wp_{j, h}\right)$, and so $e_{j k} \geq \prod_{h=0}^{k-1}\left(t_{k} M-t_{h} M\right)$ as claimed on $p$. 355, l. 9. So, inequalities (5) hold if $\Lambda_{j k} \neq \emptyset$. On the other hand, from (4) and the definition of these sets, it is easy to see that

$$
\mathbb{V}_{M} \subset \bigcup_{k=1}^{k_{0}} \bigcup_{\substack{j=1, \ldots, s_{k} \\ \Lambda_{j k} \neq \emptyset}} \mathbb{V}_{\mathbb{P}}\left(\wp_{j, k}\right) .
$$

Now, the same arguments used on $p .354, l .8-11$ give a polynomial

$$
g_{k} \in \bigcap_{\substack{j=1, \ldots, s_{k} \\ \Lambda_{j k} \neq \emptyset}} \wp_{j, k}
$$

of size $\leq c_{6} T / M$. As in $l$. 12 we put $g=\prod_{k=1}^{k_{0}} g_{k}$. Then (18) ensures that $g$ is zero over $\mathbb{V}_{M}$ and we have $t(g) \leq c_{7} T / M$. 
Unfortunately, a problem now arises in the inequality in $l .-8 /-7, p$. 362 in the proof of Theorem 2 , since (5) is available only if $\Lambda_{j k} \neq \emptyset$. This additional complication does not occur if $n=2\left(s_{1}=0\right.$ since $f$ is irreducible), so our result

$$
\tau \leq \eta+\max \left(0, \frac{4-\eta}{3}\right), \quad n=2,
$$

is still true (but it is now sharpened by Theorem 2). In the general case, however, we can easily deduce from Proposition 2 and from Theorem 1 a weak form of Theorem 2:

$$
\tau \leq \eta+\frac{n}{\eta+1} .
$$

A more precise formulation of this result is the following theorem, announced in the introduction:

TheOREM 3. For any integer $n \geq 1$ there exist constants $A, B>0$ having the following property. Let $f \in \mathbb{Z}\left[x_{1}, \ldots, x_{n}\right]$ and let $\omega$ be in the unit ball of $\mathbb{C}^{n}$. Suppose that $|f(\omega)|<\exp \left\{-C T^{\tau}\right\}$ for $C>A$ and $\tau \geq n+1$. Then we can find $\alpha \in \mathbb{C}^{n}$ on the hypersurface $\{f=0\}$ such that

$$
|\alpha-\omega|<\exp \left\{-B^{-1} C t(\alpha)^{\eta}\right\}
$$

where $\eta$ is the positive root of $\eta^{2}+(1-\tau) \eta+n-\tau=0$.

Proof. We define $M \geq 1$ as the first integer for which there exists $\lambda \in \mathbb{N}^{n}$ with $|\lambda|=M$ such that

$$
\frac{1}{M !}\left|\frac{\partial^{\lambda} f(\omega)}{\partial x^{\lambda}}\right|>-\frac{C}{2} t(f)^{\tau} .
$$

Let

$$
u=\frac{\log M}{\log t(f)} \in[0,1] .
$$

Lemma 4 gives $\alpha \in \mathbb{C}^{n}$ with $f(\alpha)=0$ and

$$
|\alpha-\omega|<\left\{-\frac{C}{4} t(f)^{\tau-u}\right\} .
$$

On the other hand, Proposition 2 with

$$
\left\{P_{1}, \ldots, P_{m}\right\}=\left\{\frac{1}{\lambda !} \frac{\partial^{\lambda} f}{\partial x^{\lambda}},|\lambda| \leq M-1\right\}
$$

and Lemma 6 of [N3] give a point $\alpha$ of multiplicity $\geq M$ on the hypersurface $\{f=0\}$ such that

$$
|\alpha-\omega|<\exp \left\{-c_{18} C t(f)^{\tau-n}\right\} .
$$

By Theorem 1, $t(\alpha) \leq c_{19} t(f) / M$, hence

$$
|\alpha-\omega|<\exp \left\{-c_{20} C t(\alpha)^{(\tau-n) /(1-u)}\right\} .
$$


Combining the last inequality with inequality (19), we find $\omega \in \mathbb{C}^{n}$ on the hypersurface $\{f=0\}$ which satisfies

$$
|\alpha-\omega|<\exp \left\{-c_{21} C t(\alpha)^{\min \{(\tau-n) /(1-u), \tau-u\}}\right\} .
$$

Since

our assertion follows.

$$
\min _{0 \leq u \leq 1} \min \left\{\frac{\tau-n}{1-u}, \tau-u\right\}=\eta
$$

\section{References}

[A] F. Amoroso, Polynomials with high multiplicity, Acta Arith. 56 (1990), 345364.

[B] W. D. Brownawell, Local diophantine Nullstellen inequalities, J. Amer. Math. Soc. 1 (1988), 311-322.

[C] G. V. Chudnovsky, Contribution to the theory of transcendental numbers, Math. Surveys Monographs 19, Amer. Math. Soc., 1984.

[G] A. O. Gelfond, Transcendental and Algebraic Numbers, Dover, New York, 1960.

[J-K-S] S. Ji, J. Kollár and B. Shiffman, A global Łojasiewicz inequality for algebraic varieties, Trans. Amer. Math. Soc. 329 (1992), 813-818.

[N1] Yu. V. Nesterenko, Estimates for the characteristic function of a prime ideal, Mat. Sb. 123 (1984), 11-34 = Math. USSR-Sb. 51 (1985), 9-32.

[N2] - On algebraic independence of algebraic powers of algebraic numbers, Mat. Sb. 123 (1984), 435-459 = Math. USSR-Sb. 51 (1985), 429-453.

[N3] - On a measure of the algebraic independence of the values of certain functions, Math. USSR-Sb. 56 (1987), 545-567.

[P1] P. Philippon, Critères pour l'indépendance algébrique, Inst. Hautes Études Sci. Publ. Math. 64 (1986), 5-52.

[P2] _, Sur les mesures d'indépendance algébrique, in: Séminaire de Théorie des Nombres, Paris 1983-84, Birkhäuser, 1985, 219-233.

[W] M. Waldschmidt, Nombres transcendants, Springer, Berlin, 1974.

DIPARTIMENTO DI MATEMATICA

VIA F. BUONARROTI 2

56127 PISA, ITALY

Received on 7.9.1992

and in revised form on 28.4 .1994 